This item was submitted to Loughborough's Research Repository by the author.

Items in Figshare are protected by copyright, with all rights reserved, unless otherwise indicated.

\title{
Chaotic transients in the switching of roto-breathers
}

PLEASE CITE THE PUBLISHED VERSION

LICENCE

CC BY-NC-ND 4.0

\section{REPOSITORY RECORD}

Giles, R.T., and F.V. Kusmartsev. 2019. "Chaotic Transients in the Switching of Roto-breathers". figshare. https://hdl.handle.net/2134/1242. 


\title{
Chaotic transients in the switching of roto-breathers
}

\author{
Richard T Giles and Feodor V Kusmartsev \\ Department of Physics, Loughborough University, Loughborough, LE11 3TU, U.K.
}

April 12, 2006

By integrating a set of model equations for Josephson ladders subjected to a uniform transverse bias current we have found almost all of the kinds of breathers described in recent experiments, and closely reproduced their voltage-current characteristics and switching behaviour. Our main result is that a chaotic transient occurs in the switching process. The growth of tiny perturbations during the chaotic transient causes the new breather configuration to be extremely sensitive to the precise history of the initial breather and can also cause the new breather to have a new centre of symmetry.

The recent discovery 1, 2, 3, of roto-breathers in Josephson ladder arrays (Fig. 1) driven by a transverse dc bias current have shown not only that these localised excitations exist but that they exhibit remarkable behaviour.

The Josephson ladder has been studied theoretically for many years. In the absence of a driving current the interaction between vortices has been found to be exponential and this leads to the vortex density exhibiting a devil's staircase as the magnetic field is increased [4, 5]. Quantum fluctuations [6], meta-stable states [7] and inductance effects [8] have been studied as has the interaction of vortices with a transverse dc bias current 9, 10.

A roto-breather 11 is a stable group of vertical junctions rotating together $\left(\theta_{j}^{\prime}-\theta_{j} \approx \omega t\right.$, see Fig. (1). Such solutions have been studied theoretically 12 in ladders driven by a uniform transverse $a c$ bias current and are also stable 13 for the case of a $d c$ bias current. Rotation at a single vertical junction was studied numerically 14,15 and two experimental groups 11, 2 have now independently confirmed the existence of such solutions and discovered further unpredicted behaviour. In an annular ladder 11] (Fig. 1 1a) and in a linear ladder 2, 3] (Fig. 1 b) breathers were observed with various numbers of "vertical" (or radial) junctions rotating. The voltage drop associated with rotating vertical junctions causes some of the horizontal junctions to rotate and various configurations were commonly observed (Fig. 2).

However the most interesting and puzzling feature was the switching between breathers as the bias current was varied. Once a breather has been initialised it is stable, but if the bias current is slowly decreased then, at some critical value of the current, the configuration of rotating junctions may change thus forming a new breather. Furthermore, it appears from the data that the new breather may have a different centre of symmetry from the old breather despite the symmetry of the ladder itself and the symmetry of the driving currents about a single vertical junction. We present here the first numerical studies of the switching behaviour of roto-breathers and show that this effect arises from the exponential growth of even infinitesimal perturbations during a chaotic transient which is associated with the switching process.

Aiming to describe the experiments as accurately as possible, we first built up a model in which the current $I$ through a junction is determined by the well-established RSJ model equation

$$
\frac{I}{I_{c}}=\frac{d^{2} \varphi}{d t^{2}}+\alpha \frac{d \varphi}{d t}+\sin \varphi
$$

where $I_{c}$ is the critical current and $\varphi=\Delta \theta-$ $\frac{2 \pi}{\Phi_{0}} \int \mathbf{A} . \mathrm{dl}$ where $\Delta \theta$ is the change in superconduct- 
ing order parameter $\theta$ across the junction and $\mathbf{A}$ is the vector potential. The "vertical" (i.e. radial) junctions may differ in area from the "horizontal" junctions by an anisotropy parameter $\eta=I_{c h} / I_{c v}=$ $C_{h} / C_{v}=R_{v} / R_{h}$ where $I_{c h}\left(I_{c v}\right), C_{h}\left(C_{v}\right)$ and $R_{h}$ $\left(R_{v}\right)$ are, respectively, the critical current, capacitance and resistance of a horizontal (vertical) junction. From Fig. 1 a we see that there are three unknowns per plaquette: $\theta_{j}, \theta_{j}^{\prime}$ and $f_{j}$. To solve for these unknowns we construct three equations per plaquette as follows. The first two equations are obtained from current conservation at the top (inner) and bottom (outer) rails. The third equation is obtained by making the approximation that the induced flux $f_{j}-f_{a}$ (where $f_{a}$ is the applied flux, assumed constant) is produced solely by the currents flowing around the immediate perimeter of the $j$ th plaquette (Fig. 11c):

$$
\frac{f_{j}-f_{a}}{\Phi_{0}}=\frac{\beta_{L}}{8 \pi}\left(I_{j+1}^{v}-I_{j}^{h}-I_{j}^{v}+I_{j}^{\prime h}\right)
$$

where $\beta_{L}$ is an inductance parameter and we have chosen $I_{c v}=1$. Eq. (2) introduces a significant difference from previous models 14, 15 which assume that the induced field is proportional to the loop (or "mesh") current circulating the plaquette. For square plaquettes, Eq. (2) represents an improvement since it properly takes into account the flux produced by the vertical conductors, although it is still not a full nearest-neighbour mutual inductance calculation 16.

Using Landau gauge we then have the following three coupled differential equations for each plaquette:

$$
\begin{gathered}
\left\{\frac{d}{d t^{2}}+\alpha \frac{d}{d t}\right\}\left(-\theta_{j-1}^{-}+4 \theta_{j}^{-}-\theta_{j+1}^{-}\right)=2 I_{j}+\sin \theta_{j-}^{-} \\
-4 \sin \theta_{j}^{-}+\sin \theta_{j+1}^{-}+\frac{8 \pi}{\beta_{L}}\left(f_{j-1}-f_{j}\right)(3) \\
\left\{\frac{d}{d t^{2}}+\alpha \frac{d}{d t}\right\}\left(\chi_{j}^{+}-\chi_{j-1}^{+}\right)= \\
\sin \chi_{j-1}^{+} \cos \chi_{j-1}^{-}-\sin \chi_{j}^{+} \cos \chi_{j}^{-}(4)
\end{gathered}
$$$$
\left\{\frac{d}{d t^{2}}+\alpha \frac{d}{d t}\right\}\left(\theta_{j+1}^{-}-\theta_{j}^{-}+2 \eta \chi_{j}^{-}\right)=
$$

$$
\sin \theta_{j}^{-}-\sin \theta_{j+1}^{-}+\frac{8 \pi}{\beta_{L}}\left(f_{j}-f_{a}\right)-2 \eta \sin \left(5_{j}\right)
$$

where $\theta_{j}^{-}=\theta_{j}^{\prime}-\theta_{j}, \theta_{j}^{+}=\theta_{j}^{\prime}+\theta_{j}, \chi_{j}^{-}=\frac{1}{2}\left(\theta_{j}^{-}-\right.$ $\left.\theta_{j+1}^{-}-2 \pi f_{j}\right), \chi_{j}^{+}=\frac{1}{2}\left(\theta_{j}^{+}-\theta_{j+1}^{+}-2 \pi F / N\right)$ and $N$ is the number of plaquettes. Putting $F=0$, this is a sufficient set of equations for describing the linear ladder (with the imposition of the appropriate boundary conditions). For the case of the annular ladder we also need one further equation for the loop flux $F$ (proportional to the total current circulating around the annulus):

$$
\frac{d^{2} F}{d t^{2}}+\alpha \frac{d F}{d t}=\frac{N^{2}\left(F_{a}-F\right)}{2 \eta B_{L}}+\frac{N}{2 \pi} \sum_{j=1}^{N} \sin \chi_{j}^{+} \cos \chi_{j}^{-}
$$

where $F_{a}$ is the applied flux, assumed constant, and $B_{L}$ is the annulus inductance parameter. Note that the equations remain invariant if all $\theta_{j}^{+}$are rotated by the same amount, an amount which can also be varied with time. It is only differences in $\theta^{+}$from one site to another which are important. Note also that the equations remain invariant if $\theta_{j}^{-}$and/or $\theta_{j}^{+}$are rotated by $4 \pi($ not $2 \pi)$.

In the RSJ model, on which our equations are based, each ideal Josephson junction has in parallel with it a capacitance and a resistance, or in other words a frequency filter. The cut-off frequency is $1 /(R C)=\alpha$. Thus voltage oscillations of frequency $\omega \gg \alpha$ tend to be suppressed, being shorted out by the capacitance. In the experiments, and in our simulations, the rotating junctions have $d \theta / d t \gg \alpha$ and so we can expect voltage oscillations to be suppressed by a large factor. We might therefore expect that all observed attractors should be characterised by having all rates of rotation, currents and fluxes at more or less fixed values. Many junctions will not rotate at all, in which case Eq.(11) gives us $I=I_{c} \sin \phi$. For those that rotate Eq.(11) gives us $I=\alpha d \phi / d t$, i.e. the supercurrent and capacitative current more or less cancel one another. We call this the $\boldsymbol{d c}$ approximation. Using this approximation one can solve for the distribution of currents, voltages and fluxes for any configuration of rotating junctions. The observed roto-breathers are in fact quite well described by this approximation. 
We now focus on determining whether or not our model exhibits the interesting switching and symmetry breaking behaviour observed in the data of Binder et al [1, 3]. Attempting to mimic the experiments, we use a random number generator to give $\theta_{j}, \theta_{j}^{\prime}$ and $f_{j}$ extremely small, but non-zero, initial values at $t=0$. If instead, the initial state were taken as having all $\theta_{j}=\theta_{j}^{\prime}=0$ then we could neglect Eq.(任) and all solutions would maintain the symmetry $\chi_{j-1}^{+}=\chi_{j}^{+}$ for all $j$; however it turns out 14] that this symmetric state can be unstable to small perturbations. Let

$$
I_{j}= \begin{cases}I_{B}+I_{\Delta} & \text { if } j=0 \\ I_{B} & \text { otherwise }\end{cases}
$$

where $I_{B}$ is called the bias current. Again following the experiment, we slowly increase $I_{\Delta}$ while keeping $I_{B}=0$ until rotation starts at site $j=0 . I_{\Delta}$ is then slowly decreased while at the same time increasing $I_{B}$ to keep $I_{0}$ constant. When $I_{B}$ has reached the desired value it is then held fixed while $I_{\Delta}$ is slowly reduced to zero. At this point, provided $I_{B}$ is not too large or small, we have just one rotating vertical junction (at $j=0$ ), the horizontal junctions rotating in the $I$-configuration (Fig. 2). We call this an $I$-breather with $N_{R}=1$ rotating vertical junctions. In Fig. 2a the $I$-breather appears to have topbottom symmetry, but in fact this symmetry is broken since $\theta^{+}$(which is more or less static) actually varies from site to site. Also the currents circulating the two plaquettes on either side of the rotating junction cause a static flux pattern rather like a vortex-antivortex pair, the value of the flux in each plaquette being what one would deduce in the dc approximation. This must cause a compressive force on the breather and an attractive force with the end of any nearby breather of the same kind.

Finally $I_{B}$ is slowly reduced while keeping $I_{\Delta}=0$. The voltage current characteristics of the breathers produced as $I_{B}$ is ramped down are shown in Fig. 3. We show here only the results for an annular ladder with $N=8$ vertical junctions and the parameter values otherwise chosen to mimic the annular ladder experiment 11, 17]. We have also put $B_{L}=0$ since we find that although the inner region of the ladder tends to pick up trapped flux (a vortex) in the breather switching process, this has little influence on the voltage-current characteristics for this range of parameter values. Note that while $I$ and $T$-breathers occur, there are no $Z$-breathers. This is because in the annular ladder the two ends of a breather are connected by a quiescent region in which all superconducting order parameters must be static or rotate together in synchrony (apart from small vibrations), and so $Z$-breathers cannot exist in the absence of other breathers.

All of the breathers are well characterised by a constant resistance $R$ (i.e. $V=\left\langle d \theta^{-} / d t\right\rangle=I_{B} R$ ) and the value of $R$ agrees very closely with the value expected in the dc approximation. Furthermore we see that at a critical rotation frequency the single-site $\left(N_{R}=1\right) I$-breather generally broadens into a multisite $I$-breather, but may also convert to a single-site or multi-site $T$-breather. In agreement with the experiments, this occurs when the rotation rate of the horizontal junctions is close to the minimum rotation rate that could be supported if the junctions were isolated and driven by a constant current (cf. the miminum rotation rate of an underdamped pendulum driven by a constant torque). This critical frequency is more or less independent of $\alpha$ (for small $\alpha$ ). The multi-site $I$-breathers switch to $T$-breathers at the same critical frequency. In the switching, two of the four rotating horizontal junctions stop while the other two double in frequency (to maintain Kirchoff's Law). As $I_{B}$ is reduced further the rotation rate of the junctions falls again until the same critical rotation rate is reached at which point all motion ceases. We obtain qualitatively similar results both for longer (and shorter) ladders and for linear ladders with similar parameter values. The main difference in linear ladders is that $Z$-breathers are produced also. These observations agree very well with the behaviour reported for a linear ladder with similar parameter values 3]. The annular ladder experiment 1] did not find any $T$-breathers but his may very well be due to a construction fault [18.

Ref. [3] shows that the linear ladder can support at least several types of breathers. Nearly all of the configurations found experimentally arose naturally in our simulations. Furthermore we found that in a multi-site breather, it appears that any two adjacent 
vertical junctions can rotate in phase or $180^{\circ}$ out of phase. It is clear that a great many breather configurations are possible.

The main result of the simulations relates to the switching to multi-site breathers. Although the single-site $I$-breather possesses left-right symmetry about $j=0$, the multi-site breather produced in the switching process frequently has a different centre of symmetry. Any breather with an even number $N_{R}$ of rotating junctions cannot have its centre of symmetry at $j=0$. Such breathers are produced even in the annular ladder (where translational invariance is exact) and even if we set all variables to zero initially, in which case the dynamical equations, initial conditions and injected currents all have exact leftright symmetry about site $j=0$. The origin of the symmetry breaking lies in the occurrence of chaotic dynamics during the switching. Further evidence of chaotic behaviour is provided by the fact that even the smallest change in initial conditions or of any parameter usually results in the production of a completely different kind of multi-site breather. Both of these effects (i.e. symmetry breaking and extreme sensitivity) were noticed in the experiments 17.

To study the origin of these effects we have looked more closely at what happens when the single-site $I$ breather becomes unstable at $V=4.3$ and switching takes place (see Fig. 3). Approaching the switching instability by reducing $I_{B}$ very slowly, we find that at $I_{B}=0.4524$ the breather appears to be stable and periodic with no positive Lyapunov exponent. However when the current is lowered to $I_{B}=0.45235$ the motion becomes weakly chaotic with a maximum Lyapunov exponent (Fig. 4 a) of $5 \times 10^{-5}$; but the chaotic motion is itself unstable and switching to a new stable breather eventually takes place after 460000 time units. Thus the switching between breathers occurs via a chaotic transient 19]. Clearly we have here the origin of the observed symmetry breaking and extreme sensitivity. The nature of the resulting final breather is extremely sensitive to the smallest change in the simulation (as one might expect given the chaotic nature of the motion) but the time before switching occurs is more or less fixed. If, before waiting for switching to occur, the bias current is slowly lowered further to 0.45234 then the maximum Lya- punov exponent increases to $2 \times 10^{-4}$ and switching occurs after only 65000 time units (Fig. $4 \mathrm{~b}$ ).

The only clear failures in the predictive power of the model occurred near the superconducting gap frequency where the RSJ model is bound to fail. In this region the experiments found that breather resistances changed and that $T$-breathers could be converted to $I$-breathers; neither of these effects were reproduced by the simulations.

We conclude that our simulations exhibit most of the main features of the roto-breathers recently observed in Josephson ladder arrays. In addition to reproducing the experiments we have found that switching occurs via a chaotic transient, and this may be a general feature of the switching of roto-breathers in Josephson arrays. This chaotic transient has some practical consequences. First of all it leads to the extreme sensitivity and symmetry breaking already observed in the experiments. Secondly, since it has has a very broad Fourier spectrum, characteristic of chaotic motion, we can predict the existence of a source of noise associated with switching. The duration of the noise (i.e. the transient) should depend on how quickly the bias current is ramped (Fig. 14). We hope that future experiments will discover the noise and confirm this effect.

We are grateful to A.V. Ustinov and P. Binder for kindly giving us their data and explaining it prior to publication. We are also grateful for discussions with M.V. Fistul, S. Flach, A. Osbaldestin and J.H. Samson and for the hospitality of the Max-Planck-Institut, Dresden and the Universität Erlangen-Nürnberg.

\section{References}

[1] P. Binder et al., Phys. Rev. Lett. 84, 745 (2000).

[2] E. Trías, J. Mazo, and T. Orlando, Phys. Rev. Lett. 84, 741 (2000).

[3] P. Binder, D. Abraimov, and A. Ustinov, Phys. Rev. E 62, 2858 (2000).

[4] M. Kardar, Phys. Rev. B 30, 6368 (1984). 
[5] R. Giles and F. Kusmartsev, J. Low Temp. Phys. 117, 623 (1999).

[6] M. Kardar, Phys. Rev. B 33, 3125 (1986).

[7] J. Mazo, F. Falo, and L. Floría, Phys. Rev. B 52, 10433 (1995).

[8] J. Mazo and J. Ciria, Phys. Rev. B 54, 16068 (1996).

[9] S. Kim, Phys. Lett. A 235, 408 (1997).

[10] M. Barahona, S. Strogatz, and T. Orlando, Phys. Rev. B 57, 1181 (1998), and references therein.

[11] S. Takeno and M. Peyrard, Physica D 92, 140 (1996).

[12] P. Martinez, L. Floría, F. Falo, and J. Mazo, Europhys. Lett. 45, 444 (1999).

[13] R. MacKay and J.-A. Sepulchre, Physica D 119 148 (1998).

[14] J. Mazo, E. Trías, and T. Orlando, Phys. Rev. $B$ 59, 13604 (1999).

[15] S. Flach and M. Spicci, J. Phys.: Condens. Matter 11, 321 (1999).

[16] D. Domínguez and J. José, Phys. Rev. B 53, 11692 (1996).

[17] P. Binder, private communication.

[18] See footnote 14 of Ref. [3].

[19] R. Hilborn, Chaos and Nonlinear Dynamics (Oxford, UK, 1994).
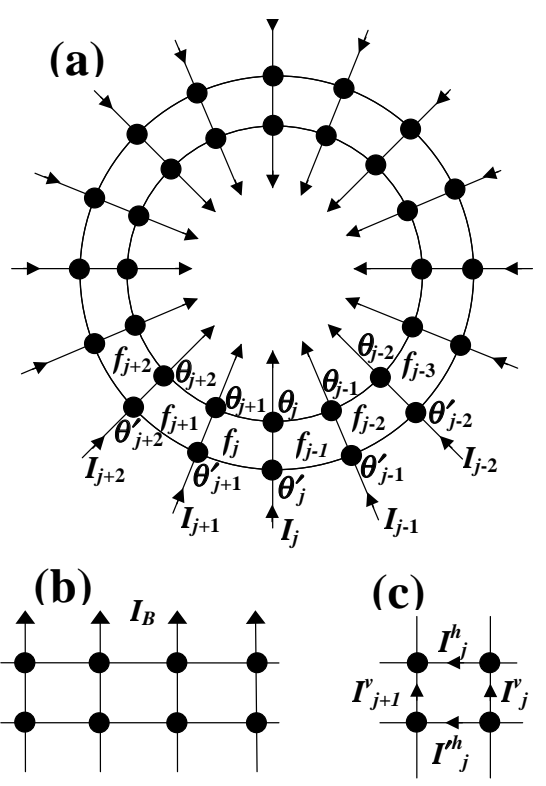

Figure 1: (a) An annular ladder subjected to transverse bias currents $I_{j}$. Each circle represents a superconducting island. Each link between islands represents a Josephson junction. $\theta$ is the phase of the superconducting order parameter and $f$ is the flux threading a plaquette. (b) A linear ladder. (c) Explanation of the notation used in Eq. (2).

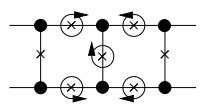

(a)

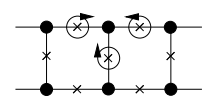

(b)

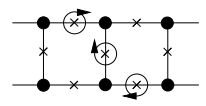

(c)
Figure 2: Three configurations for the rotation of horizontal junctions around a breather with $N_{R}=1$ vertical rotating junctions: (a) the $I$-breather, (b) the $T$-breather, and (c) the $Z$-breather. 


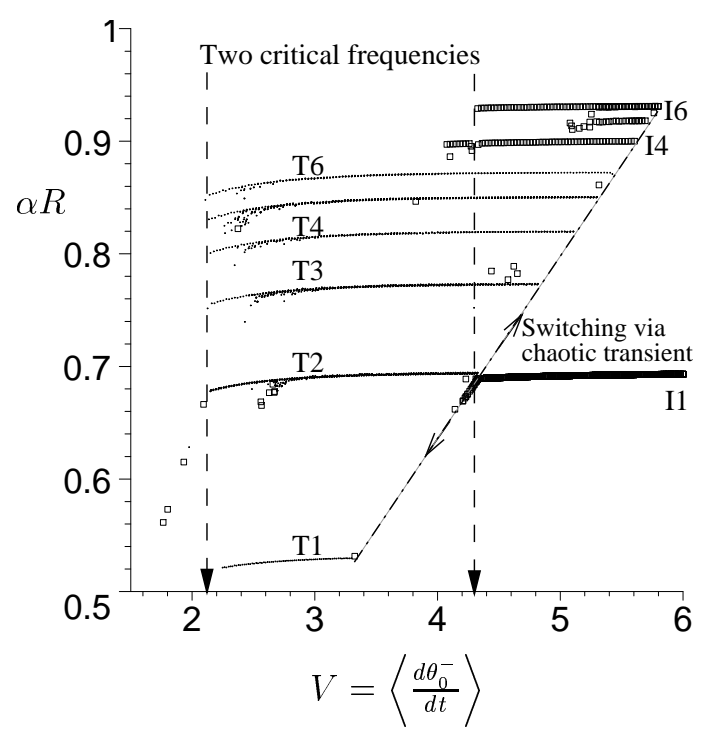

Figure 3: Resistance $\alpha R$ (where $R=V / I_{B}$ ) plotted against voltage $V=\left\langle d \theta_{0}^{-} / d t\right\rangle$. The results were obtained for an annular ladder with $N=8$ vertical junctions, $\alpha=0.07, \eta=0.44, \beta_{L}=4.8$, $B_{L}=F_{a}=f_{a}=0$. The middle vertical junction was excited in the manner described in the text and then the uniform bias current $I_{B}$ was ramped down. The data come from many runs with different starting values of $I_{B}$. The labels T2, I1 etc. refer to breather types, e.g. "I1" means the one-site $I$-breather.

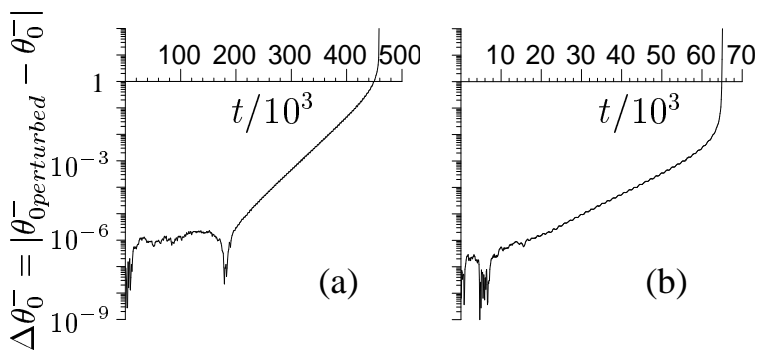

Figure 4: Exponential growth of perturbations during the chaotic transient which occurs when the single-site $I$-breather becomes unstable at $V=$ $\left\langle d \theta^{-} / d t\right\rangle=4.3$. (a) The initial state $(t=0)$ was prepared with $I_{B}=0.45235$. A perturbed initial state was then produced by introducing small random changes to all state variables. After an initial period $\Delta \theta_{0}^{-}$starts to grow exponentially with time. Switching to a new stable breather takes place at $t=460,000$ and produces the sudden rise in $\Delta \theta_{0}^{-}$ at that time. (b) Same except the initial state was prepared with $I_{B}=0.45234$. 